\title{
The Research and Application of Intelligent Broadcast System of Coal Mine
}

\author{
Xiucai Guo, Xiaomin Lu \\ Institute of electrical and control engineering Xi'an University of science and technology, Xi'an 710054, China
}

\begin{abstract}
This paper briefly describes the practical significance of coal mine broadcasting system, a comprehensive consideration of functional requirements and the control demands of the system, analyzing the advantages of Industrial Ethernet combines CAN field bus technology and the feasibilities of it's applications in the intelligent coal mine broadcasting system, we design a bidirectional intelligent broadcasting system can be used in mine communication system. This paper, illustrates the basic structure of system, analyzes the real-time of CAN bus, proposes hybrid scheduling algorithms, and designs the gateway node program finally. The system of low cost, good openness, extension, installation, and maintenance are convenient, advanced functions, can meet the needs of the mine emergency communication.
\end{abstract}

Keywords-industrial ethernet; CAN bus; TCP/IP; broadcasting.

\section{INTRODUCTION}

Coal is China's important energy, coal mine mining, but at the same time, coal mine work also has very high risk, the underground safety accidents seriously threaten the safety of mine workers. In recent years, our country improves the safety situation of the coal mine production, especially, the computer, communication and control technology of overlapping gradually application in the intelligent broadcasting system; it makes outstanding contributions to curbing production of coal mine safety accidents.

Coal mine broadcast has a big power also real-time broadcasting radio system, so the information of language communicates quickly, can finish all kinds of scheduling, the production, the safety instructions issued in the first place, and the communications network has no paralysis and congestion, so it becomes an important communication guarantee, greatly improving the capacity of the disaster resistance and disaster prevention. At present, our traditional coal mine radio system used the transmission way exist the following problems: no advanced function enough; transfer rate is slow; Real-time is bad; it alao has a short transmission distance as a high speed transmisson; a poor expansibility of system; network mode is not flexible; weak anti-interference ability; information integration ability is not strong and so on. Therefore, in order to realize the underground radio communication smoothly, ensure that under the condition of sudden disaster in mine, the persons underground evacuate in the first time, so as to minimize personnel and financial losses, achieve the goal of safe protection, we must design a safe, stable, easy to use, easy maintenance, extensible, and advanced mining radio communication system in the special environment of the mining production.

\section{THE SCHEME OF SYSTEM}

Due to the special environment of mine, in the actual production, often requires a point that when an accident occurs, the node can be as a radio source, to send out alarm signal to the other location and the ground control center in time, which requires the control node have a bit of multipoint control function. Based on these characteristics, , using a network system architecture which based on industrial Ethernet standard TCP/IP that combines CAN field bus, to complete the design of the mine intelligent broadcasting system after comparing with the traditional schemes. It mainly based on the following considerations.

i) Industrial Ethernet technology has been used under mine, which transmites through fibre-optical, it has some advantages that a good openness, high data transmission rate, and easy to integrate network, the key is to use interchangeable Ethernet technology for controller and operation station, the coordinates and cooperations among the various workstations which provides an interactive mechanism, and integrates the upper information network seamlessly. However, in the process of a practical application of mine which based on Industrial Ethernet IP network broadcasting mode has some problems, mainly reflected in:

Small capacity: Although IP broadcasting extends easily, as a result of using IP broadcasting way occupies a large number of IP resources of coal mine local ring network, which causes that IP broadcasting system capacity is limited.

High cost: Every radio site needs to be connected to the Industrial Ethernet ring network, along with the extension of the mine mining areas, the positions of each monitoring node needs to be changed constantly, at this time, if only uses the way of fibre-optical transmission, we will need changing the length of fiber-optical constantly; When the radio station in working areas disappears from working areas, the fiber-optical won't be used again, which causes a waste of fibre-optical resources.

ii) The coal mine intelligent broadcasting system based on CAN bus Technology design is safe and reliable in the aspects such as networking, wiring, installation and maintenance, it has obvious advantages which compared with RS-485 bus technology that in the past whose characteristics such as the master node, deal closed, universal property is bad, low transmission rate, has a bad real-time characteristics under the environment of multiple 
parameters and large amount data. CAN bus works using multiple main stations and the bus can carry 110 nodes at most, so all the functional modules of the system CAN be directly attached to the bus. And also solved the problem of using Ethernet alone is not convenient to voice and data transmission control, so CAN bus can be used in radio substation well.

iii) Applying Industrial Ethernet and CAN bus well, can show all their different kinds of functions that the formation of complementary advantages, improve the overall system performance, to achieve seamless integration.

This topic has designed a set of interactive two-way based on Industrial Ethernet and CAN bus IP radio dispatch system, solving the low efficiency of using CAN bus transmission separately. CAN bus is chosen to transmission data underground substation, will CAN Ethernet module for underground broadcasting system, the actual mining cables is replaced, making full use of existing network resources underground, extending transmission distance of the signal effectively. On the basis of the original IP broadcast, will become every IP sites into data substation of broadcast system, at the same time of expanding the system capacity of broadcasting system, also changes transfer medium that fiber-optical into a twisted pair, reduces the system cost.

\section{THE FUNCTION OF THE SYSTEM}

The system uses the way of propaganding broadcast and playing music, which plays an important role in the daily production and management, soothing emotions of the mine personnel; When sudden accidents occurs, it plays a guide to evacuate the distress mine personnel timely[1]. Mainly realize the functions of this system are: regular radio, partition, emergency broadcast radio, emergency rescue, site monitoring, file downloads, microphone radio, condition monitoring, history inquiry, security monitoring, remote control, phone radio.

\section{SYSTEM WORKING PRINCIPLE AND BASIC STRUCTURE}

\section{A. Working principle of the system}

Control instructions and the audio data are encapsulated as IP packets which transfers to date substation through fiber-optical ring network in the process of the whole system, after the station receiving the packets and parse them, then it segregates the date according to the analytical data, encapsulates it in the standard of the CAN data frames, and then through the field bus to transfer the data to the specified radio substation data. Radio terminal substation perform receives the control commands then play the audio data. Also through radio stations for emergency calls, realizing a two-way radio function between it and control cabin, reporting the situation immediately in the region. So this system uses a double address addressing method for data transmission, the IP addressing combined with CAN way of addressing.

\section{B. Basic architecture of the system}

The system based on Industrial Ethernet and CAN bus intelligent radio communication system is mainly composed of ground spread broadcast equipment and downhole phone and spread broadcast voice box, The whole system can be roughly divided into three parts: control center, data substation and radio substation. Its architecture diagram is shown in figure 1.

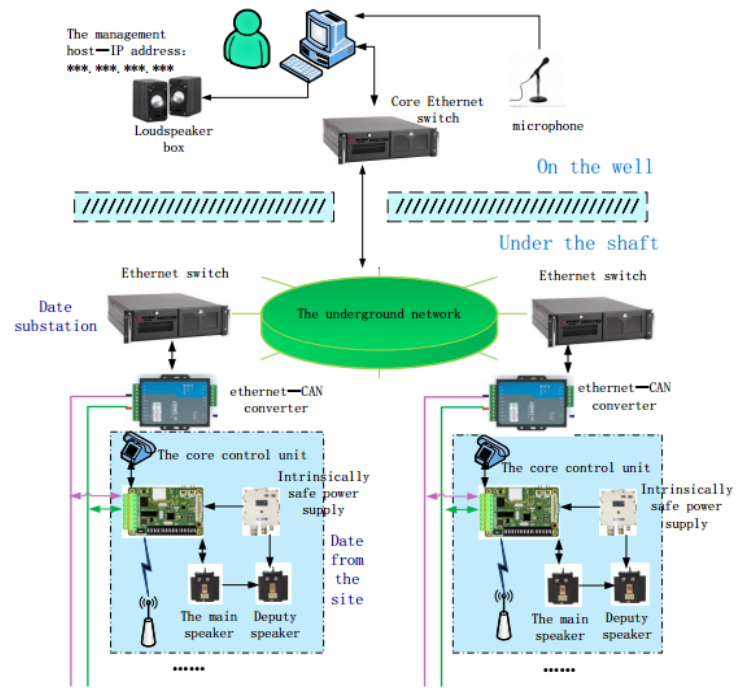

Figure 1. System architecture’s diagram

\section{1) The control center}

The broadcasting system control center is equipped and managed by the host (industrial PC) which is installed mine intelligent control software. Outside is equipped with a microphone, alarm speakers, UPS power supply and other supporting facilities. The main control server of the system provides system management, user management, group management and installation management. it manages the entire broadcasting system through coal inoue intelligent radio management software when it works, finally realizes the function that unattended and intelligent control of broadcasting system.

\section{2) Data substation}

Data substation is freight station that all the broadcasting system communicates responsible for protocol conversion. This section uses the development platform which regards ARM7chip LPC2378 as the core, uses high-speed transceiver TJA1050; it connects MCU through SPI (Serial Peripheral Interface). Ethernet controller adopts high speed Ethernet interface chip DM9000 which in DAVICOM company. System external SDRAM and FLASH as the medium of system memory space and file systems. The gateway hardware structure diagram is as shown in figure 2 .

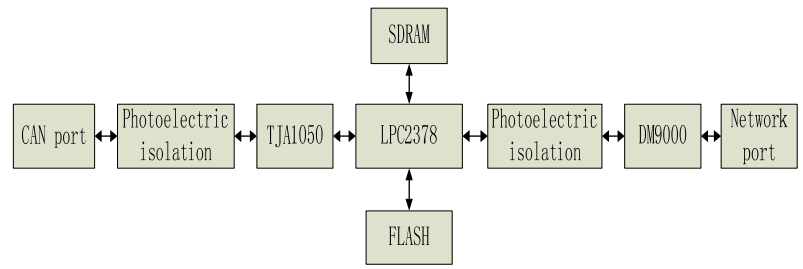

Figure 2. System structure of the TCP/IP-CAN protocol conversion 


\section{3) Radio substation}

Radio substation is the terminal of the broadcasting system, it and data substation are interdependent, radio substation can only at a certain specified data substation, cannot be directly connected to Industrial Ethernet ring network, process data sent by data substation. Radio substation, consists of main speakers, expanding on the telephone, the module of wireless transceiver and several ancillary speakers. The core of the control unit extends the wireless transceiver module to make the combination of wired and wireless. The main sound box is core control unit of broadcasting substation; The main speakers can realize full duplex two-way voice between broadcast substation and control center or other sites; Ancillary speakers and the main speakers series, so it expands the scope of broadcasting, also facilitate extension. The main speaker and ancillary speaker are independent of the power supply.

\section{THE ANALYSIS AND RESEARCH OF THE SYSTEM OF CAN BUS REAL-TIME}

The most important factors of influence CAN bus realtime transmission are transfer time and waiting time. Transmission time is mainly determined by the baud rate and transmits digits. The length of the CAN bus as shown in type 1 ,

$$
\mathrm{g}+8 \mathrm{~L}+13
$$

$\mathrm{g}$ is 34When the message is the standard frame and $\mathrm{g}$ is 54 to extension frames. CAN bus is adopted a filling method for encoding, In the bit stream as long as there are five consecutive the same value, automatically inserting a value instead of padding. Frame start, arbitration and control field, data field and CRC sequence are encoded by using the method of filling, which participate in filling a total of g+8L. So the maximum value of the total length for a message is as shown in type 2 .

$$
\mathrm{g}+8 \mathrm{~L}+13+\left\lfloor\frac{8 L+\mathrm{g}-1}{4}\right\rfloor
$$

Therefore, the transmission of a message length can be unpredictable, so shorten the waiting time of packet can improve the real-time performance of the bus. A good scheduling algorithm can ensure that as much information as possible in the transmission to complete before the deadline.

In a coal mine broadcasting system, the information of CAN bus transmits is divided into four types; they are emergency hard real-time, ordinary hard real-time, soft realtime information, and non-real-time information. Hard realtime information refers to the broadcasting system in various sudden information, such as alarm information, scheduling information; Soft real-time information refers to the periodic control information; Non real-time information refers to the configuration data, part of the system status monitoring information [2].

CAN bus have good real time, but it produces blocking phenomenon when the load becomes heavier, make the low priority data deadlocks. In view of this, the topic proposed hybrid scheduling algorithm, and it is shown in figure 3 .

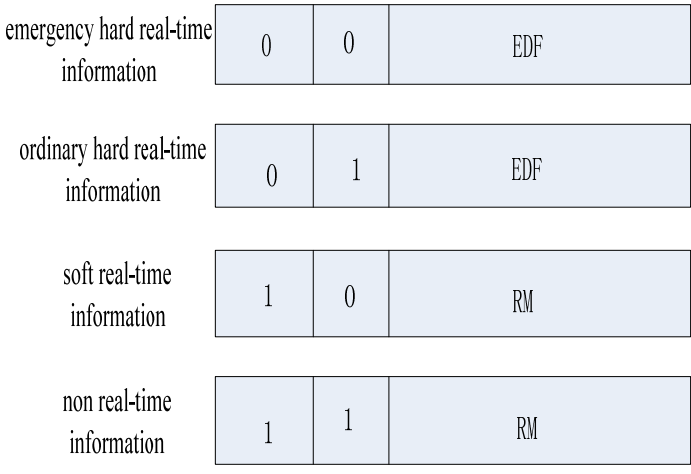

Figure 3. Hybrid scheduling model diagram

Set the first place of hard real-time information ID to 0 at first, so, the priority is higher than the soft real-time and non-real-time information, set the second place of ordinary hard real-time information ID to 1 . Hard real-time message use dynamic EDF scheduling algorithm to guarantee delivery of key information in a timely manner. Set the first place of soft real-time information and the first two place of non-real-time information ID to1 and uses the optimal periodic messages static RM scheduling algorithm. Hybrid scheduling algorithm, the advantages of static scheduling and dynamic scheduling, ensure the priority sequence of the system and improve the real-time performance.

\section{GATEWAY NODE PROGRAM OF THE SYSTEM}

The system is as example as the gateway node, detailed in the work process node, as shown in figure 4 for the gateway node of the main program.

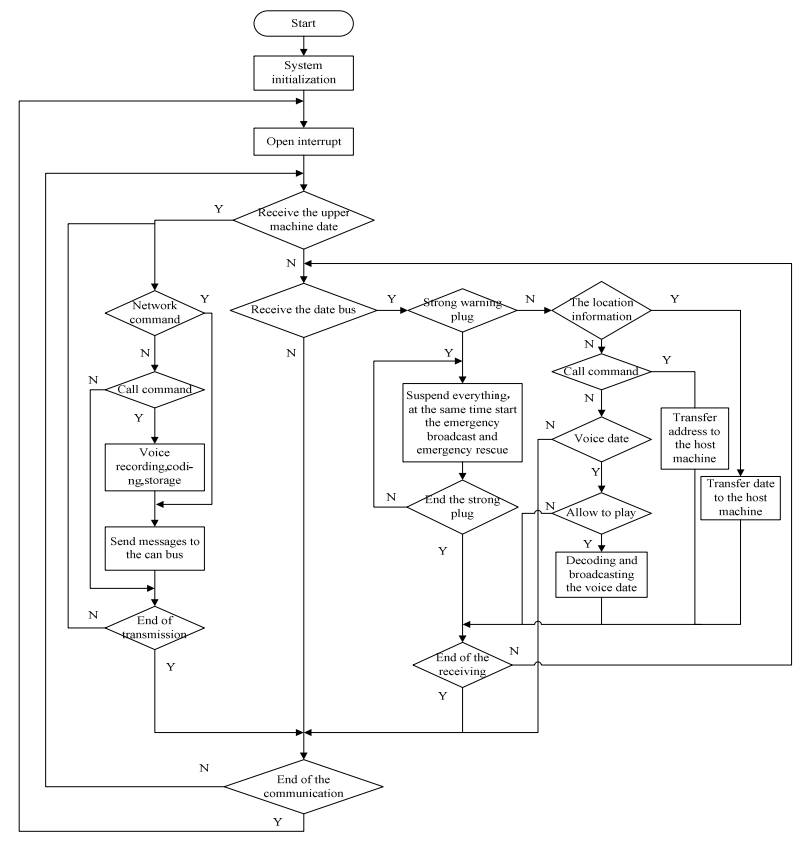

Figure 4. The main program flow chart of gateway node 
i) The gateway nodes receive electricity and initialization. Initialization processes include the SJA1000 initialization, UART initialization and I/O serial port initialization. Then the node open interrupt, waiting for receiving data;

ii) When the gateway nodes receive the upper machine, storage control information and the target address, and determine what kind of command. If the network information, control and network address information is sent to the CAN bus; If need to send voice messages, to pass through the recording, the voice signal coding and the destination node address together after sent to CAN bus [3].

iii) If the gateway node receives the data from CAN bus, judgments the types of information at first. If strong thrust for fire alarm reporting, stop all, then start the emergency broadcast and emergency assistance. If for the node location information, the information transmitted and stored up a machine. If the call command, first request to the address of the node into the PC and rang the bell, showed a node call $\mathrm{PC}$, judge whether answer or not; If for voice and data, the judgment whether to play.

iiii) When a group of data receiver-processing-send ended reset the MCU, the re-initialization, waiting for the next round of communication.

\section{CONCLUSIONS}

The design of coal mine intelligent broadcast system which based on Industrial Ethernet and CAN bus technology is safe and reliable in some aspects such as networking, wiring, installation and maintenance, CAN bus technology gives consideration to the real-time and transmission distance of control, improves the data transmission distance on the premise of guarantee real-time requirements[4]. And for not wiring corner uses the nearest terminal controller to extend radio control wireless modules. Of course, the system also has some flaws, CAN bus scheduling needs to be improved constantly.

\section{REFERENCES}

[1] Huang X R,Gu W L. The design and implementation of coal mine voice emergency broadcasting system [J]. Computer knowledge and technology, 2014,04:850-852.

[2] Qiao Y. Body CAN control network based on hybrid scheduling algorithm research [D]. Jilin university, 2013.

[3] Peng X. The underground communication system based on CAN bus [D]. Chongqing: Chongqing university.2013

[4] Yang J F. Field bus technology in the application of the coal mine safety monitoring system [J].Science and technology entrepreneurs,2013,11:97. 